\title{
Nonlinear filtering based on 3D wavelet transform for MRI denoising
}

\author{
Yang Wang ${ }^{1 *}$, Xiaoqian $\mathrm{Che}^{2}$ and Siliang $\mathrm{Ma}^{1}$
}

\begin{abstract}
Magnetic resonance (MR) images are normally corrupted by random noise which makes the automatic feature extraction and analysis of clinical data complicated. Therefore, denoising methods have traditionally been applied to improve MR image quality. In this study, we proposed a 3D extension of the wavelet transform (WT)-based bilateral filtering for Rician noise removal. Due to delineating capability of wavelet, 3D WT was employed to provide effective representation of the noisy coefficients. Bilateral filtering of the approximation coefficients in a modified neighborhood improved the denoising efficiency and effectively preserved the relevant edge features. Meanwhile, the detailed subbands were processed with an enhanced NeighShrink thresholding algorithm. Validation was performed on both simulated and real clinical data. Using the peak signal-to-noise ratio (PSNR) to quantify the amount of noise of the MR images, we have achieved an average PSNR enhancement of 1.32 times with simulated data. The quantitative and the qualitative measures used as the quality metrics demonstrated the ability of the proposed method for noise cancellation.
\end{abstract}

Keywords: magnetic resonance imaging, 3D image denoising, 3D wavelet transform, bilateral filtering, enhanced NeighShrink thresholding

\section{Introduction}

Three-dimensional magnetic resonance imaging (MRI) has, during the last several decades, benefited from a variety of technological developments resulting in increased resolution, signal-to-noise ratio (SNR), and acquisition speed. However, fundamental trade-offs among resolution, acquisition speed, and SNR combined with scientific, clinical, and financial pressures to obtain more data more quickly, can result in images that exhibit significant artifacts, e.g., noise, partial volume, and intensity nonuniformity. For instance, the need for shorter acquisition times for patients in certain clinical studies often undermines the ability to obtain images having both high-resolution and high SNR. Another example concerns diffusion-tensor (DT) MRI that has become quite popular over the last decade due to its ability to measure the anisotropic diffusion of water in structured biological tissue. DT MRI differentiates between the anatomical structures of cerebral white

\footnotetext{
* Correspondence: wyangjlu@gmail.com

'Department of Computational Mathematics, Jilin University, Chang chun, China

Full list of author information is available at the end of the article
}

matter, which was previously impossible with MRI, in vivo, and noninvasively. The effects of Rician noise on DT MRI, however, are severe because of the inherent nature of the process-higher tissue anisotropy produces progressively lower intensities in diffusion-weighted images that, in turn, are more susceptible to Rician noise. The efficacy of higher-level post processing of MR and DT-MR images, e.g., segmentation and registration, that assume specific models on regions of interests, e.g., homogeneous, is sometimes impaired by even moderate noise levels. Hence, it is necessary to remove the noise from MR image.

The removal of noise from noisy data to obtain the unknown signal is often referred to as denoising. Postprocessing filtering techniques with the advantage of not to increase the acquisition time have extensively been used in MRI denoising. Many image denoising methods have been proposed in previous research. The conventional approach $[1,2]$ was proposed to estimate the Rician noise level and perform signal reconstruction using a maximum likelihood method. Anisotropic diffusion [3-5] reduces image noise by considering a scale space, and it has been adapted to suppress the Rician 
noise in MR image [6]. Moreover, anisotropic diffusion filter combined with the Wiener filter [7] has been used for MRI denoising, which spatially averages pixels according to their correlation structure. The nonlocal means filter has been applied for feature preserved MRI denoising [8-12]. It builds an estimation of the restored pixel value by weighted averaging over a large portion of the pixels within the image. The weights are based on the similarity computed by comparing the patches instead of single point, and the edges and the details can both be well preserved.

Recently, wavelets have become a popular tool in various applications for data analysis and image processing. Application of wavelets for denoising of MR images has produced a large number of algorithms $[13,14]$. Early study [15] was followed by multi-scale products thresholding [16], which uses adjacent wavelet subbands to detach the edges from noise. Complex denoising of MR images using wavelets was proposed by Zaroubi and Goelman [17]. The method produces better SNR compared to the magnitude denoising scheme. Wu et al. [18] proposed a wavelet-based background noise removal method in MRI. The proposed method can be used jointly with existing denoising methods to improve their effectiveness. Bilateral filtering in wavelet domain has been shown to preserve the edges efficiently [19]. Moreover, wavelet has been used for MRI denoising in combination with Radon transform, which estimates noise variance in different scales [20].

In this study, we proposed a $3 \mathrm{D}$ extension of the wavelet transform (WT)-based bilateral filtering ideas for Rician noise removal. Due to delineating capability of wavelet, 3D WT was employed to decompose the MR image into the approximation and the detailed subbands. Next, bilateral filtering of the approximate coefficients in a modified 3D neighborhood improved denoising efficiency and effectively preserved relevant edge features. Meanwhile, the detailed subbands were processed with a weighted NeighShrink (WNS) thresholding algorithm. At the end, inverse 3D WT was performed on the selected subbands to obtain final denoised image. In the proposed method, the combined property of 3D WT and the bilateral filter significantly reduces the blurring of image features.

The structure of this article is as follows. First we describe our proposed noise cancellation algorithm (Section 2). Then, we explain our experimental methodology and present the results with both synthetic and real images (Section 3). Finally, Section 4 is devoted to discussion and conclusion.

\section{Materials and methods}

\subsection{Rician noise estimation}

One main source of noise in MRI signal is the thermal noise. The signal component of the measurement is present in both real and imaginary channels; each of the two orthogonal channels is affected by white Gaussian noise. An MR image is usually reconstructed by computing the inverse discrete Fourier transform of the raw data. The magnitude image of the reconstructed MRI is used for visual inspection and for automatic computer analysis. Since the magnitude reconstruction is the square root of the sum of two independent Gaussian random variables, the magnitude image data are described by a Rician distribution [21].

The complex MRI data is given as

$$
y=p+i q
$$

The noise in the complex raw data is zero mean Gaussian noise and the spatial MR image is the magnitude of the noisy raw data. Therefore, the magnitude of the noisy raw data $z$ is given by

$$
\begin{aligned}
& z=\sqrt{\left(p+n_{r e}\right)^{2}+\left(q+n_{\text {im }}\right)^{2}} \\
& n_{\mathrm{re}}, n_{\mathrm{im}} \sim G\left(0, \sigma^{2}\right)
\end{aligned}
$$

i.e., $z$ is corrupted by Rician noise. Under these conditions, it is advantageous to take the square of the magnitude MR image. Its expectation reads

$$
E\left(z^{2}\right)=E\left(\gamma^{2}\right)+2 \sigma_{n}^{2}
$$

And we could obtain an unbiased estimator of $y$ by taking

$$
y \approx \sqrt{\max \left(0, z^{2}-2 \sigma_{n}^{2}\right)}
$$

where the maximum function is applied to avoid physically meaningless complex values. Note that this unbiasing procedure relies on a proper estimate of the $\sigma_{n}$ parameter. To this end, many methods have previously been reported [22-25]. They are mainly based on the features of the Rayleigh background, for instance,

$$
\sigma_{n}=\sqrt{\frac{\mu}{2}}
$$

where $\mu$ is the mean value of the background of the squared magnitude of image, these methods are suitable for our method as long as the MR image contains background. 


\subsection{D WT}

Wavelets are orthogonal basis functions that delve data into different spatio-frequency components. The delineating capability of wavelet leads to better discrimination between the noise and the signal. In terms of wavelet space decomposition, the separable 3D WT [26] can be expressed by a tensor product by

$$
\begin{aligned}
V^{3}= & \left(L^{x} \oplus H^{x}\right) \otimes\left(L^{y} \oplus H^{y}\right) \otimes\left(L^{z} \oplus H^{z}\right) \\
& =L^{x} L^{y} L^{z} \oplus L^{x} H^{y} L^{z} \oplus H^{x} L^{y} L^{z} \oplus H^{x} H^{y} L^{z} \\
& \oplus L^{x} L^{y} H^{z} \oplus L^{x} H^{y} H^{z} \oplus H^{x} L^{y} H^{z} \oplus H^{x} H^{y} H^{z}
\end{aligned}
$$

where $\oplus$ denotes space direct sum, $L^{\alpha}$ and $H^{\beta}$, respectively, represent the high- and low-pass directional filters along directions of $\alpha$-axis, where $\alpha \in\{x, y, z\}$.

Figure 1 shows a separable 3D decomposition of a volume: after being applied on the rows and on the columns, the analysis filters followed by a 2 to 1 decimation are applied along the third dimension. After the decomposition, eight subvolumes of lower resolution were obtained: the approximation subvolume from resolution ' -1 ' named $L^{x} L^{y} L^{z}$ and 7 subvolumes of details. The separable $3 \mathrm{D}$ wavelet provided an equal decorrelation of the original volume voxels in the three directions and the wavelet-based denoising was achieved by modifying the contents in the octant subbands, followed by wavelet synthesis.

\subsection{D bilateral filtering}

For 2D image denoising, the original 2D bilateral filter [27] computes the similarity of two pixels by comparing the similarity of the two pixels' square neighborhood centered at the pixels. In a similar way, the similarity of two voxels in $3 \mathrm{D}$ image is determined by comparing the similarity of their cubic neighborhood centered at the voxels. For a given size, the cubic neighborhood contains more voxels than the square one. Thus, even for a small neighbor size, some voxels similar to the voxel being processed in a certain extent have low weight when using $3 \mathrm{D}$ neighbors. But, if $2 \mathrm{D}$ square neighbor is

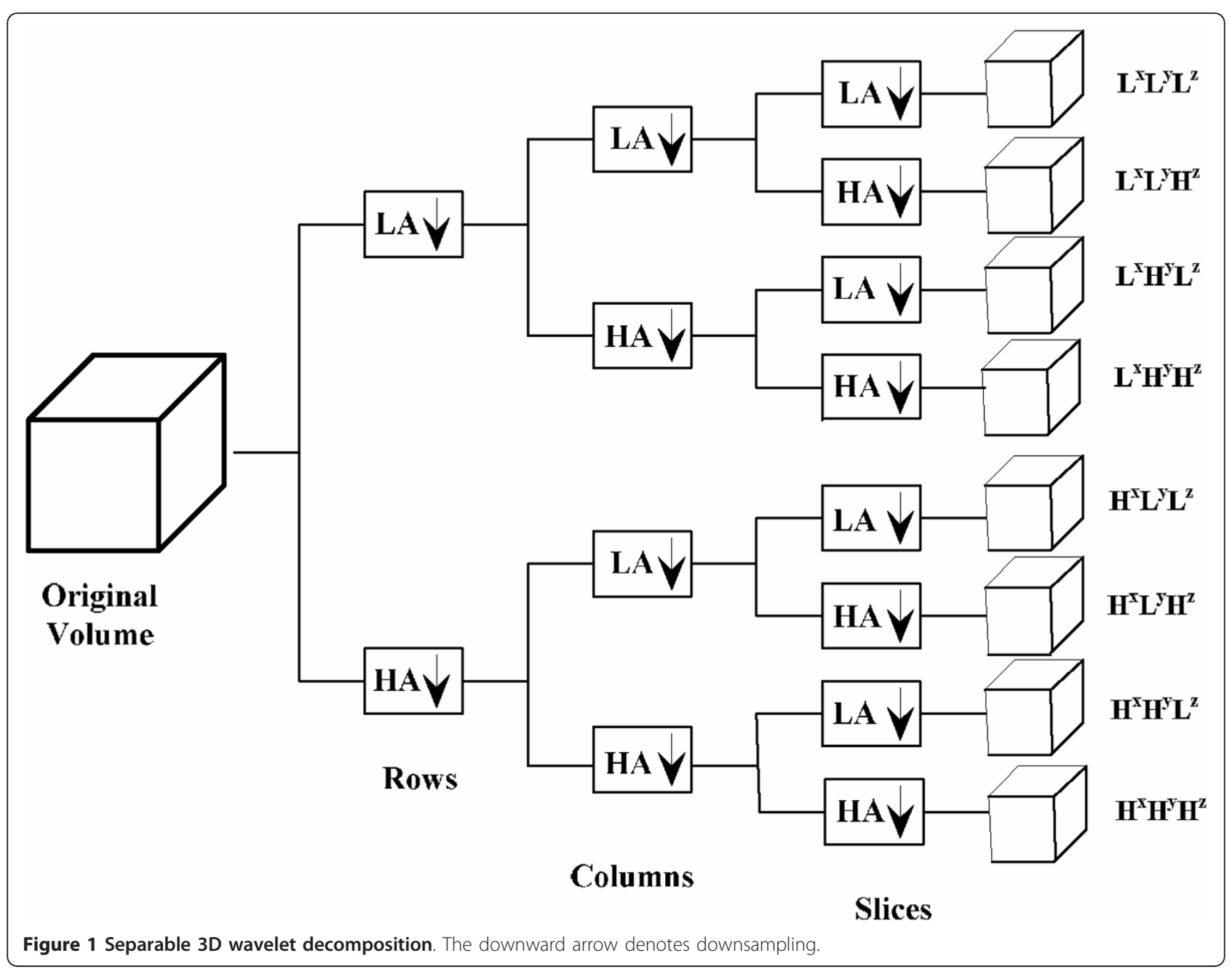


used to compare similarity in 3D images, many voxels not very similar to the voxel being processed will involve in the decision of the new value. To address this issue, we employed a new neighbor for computing the similarity of two voxels. The modified neighborhood consisted of one square in a plane and one line in another axis that was normal to the plane. The square neighbor and the line were both centered at the voxel being studied, as shown in Figure 2.

The neighborhood that consists of a square and a line contains part of the 3D structure of the voxel's neighborhood and contains fewer voxels than the cubic neighbor. Thus, more similar voxels could be identified. The 3D bilateral filter with improved neighborhood is as follows:

$$
\begin{aligned}
& f(i, j, k)=\frac{1}{C} \sum_{(p, q, m) \in O(i, j, k)} w_{d(i, j, k)}(p, q, m) \cdot w_{r(i, j, k)}(p, q, m) \cdot g(p, q, m) \\
& w_{r(i, j, k)}(p, q, m)=\exp \left(-\frac{|g(p, q, m)-g(i, j, k)|^{2}}{2 \delta_{r}^{2}}\right) \\
& w_{d(i, j, k)}(p, q, m)=\exp \left(-\frac{\left|(p-i)^{2}+(q-j)^{2}+(k-m)^{2}\right|}{2 \delta_{d}^{2}}\right) \\
& C=\sum_{(p, q, m) \in O(i, j, k)} w_{d(i, j, k)}(p, q, m) \cdot w_{r(i, j, k)}(p, q, m)
\end{aligned}
$$

where $g(p, q, m)$ represents the intensity value of voxel at position $(p, q, m)$ of volume, $\mathrm{O}(i, j, k)$ represents the modified neighborhood of voxel at position $(i, j, k), f(i, j$, $k$ ) represents the filtered value, $w_{d}$ and $w_{r}$ are spatial and radiometric components of the bilateral filter,

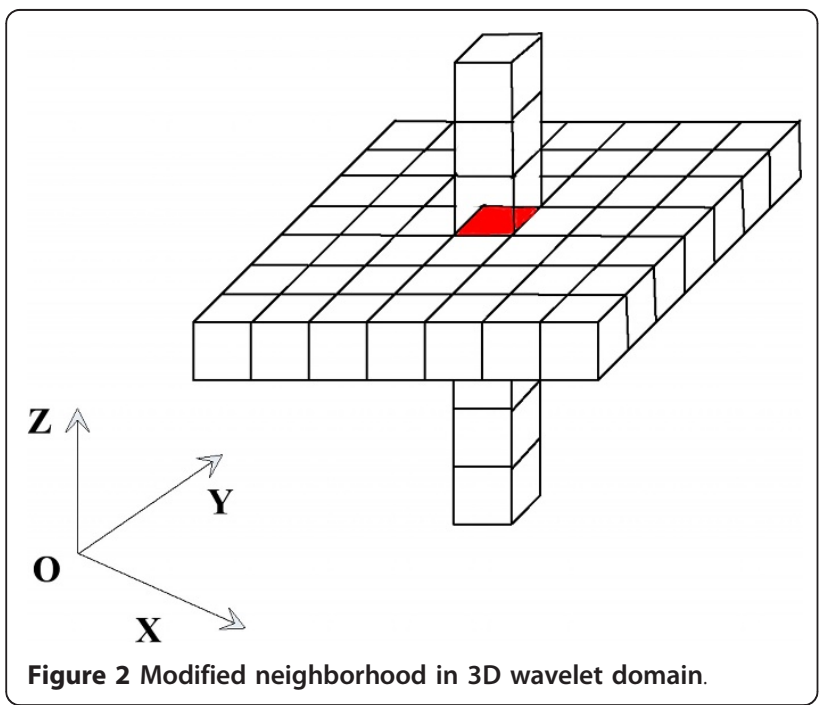

respectively, the parameters $\delta_{d}$ and $\delta_{r}$ control the behavior of the weights.

\subsection{D WNS thresholding}

Recent study on wavelet thresholding evolves as block processing, in which the coefficient is most likely to contain signal if its neighborhood also contains signal coefficient. This method is called NeighShrink [28]. Zhou and Cheng [29] have improved it by optimally choosing the NeighShrink parameters based on Stein's unbiased risk estimate (SURE). We extended the Neighshrink algorithm into 3D domain as following:

For the 3D wavelet $w_{i j k}$ coefficient to be shrunk, consider a cubic neighborhood $B_{i j k}$ centered at $w_{i j k}$, as shown in Figure 3. The size of neighborhood is represented as $3 \times 3 \times 3$. For $S_{i j k}^{2}=\sum_{(p, q, m) \in B_{i j k}} w_{p q m}^{2}$, the NeighShrink shrinkage formula is given by [29]

$$
\widehat{\theta}_{i j k}=w_{i j k} \max \left(1-\frac{\lambda^{2}}{S_{i j k}^{2}}, 0\right)
$$

where $\hat{\theta}_{i j k}$ is the estimator of the unknown noiseless coefficient and $\lambda$ is the threshold. The optimal $\lambda$ for each of the high-frequency subbands is estimated using SURE.

In the Neighshrink method, all the wavelet coefficients are shrunk to achieve the purpose of denoising. The

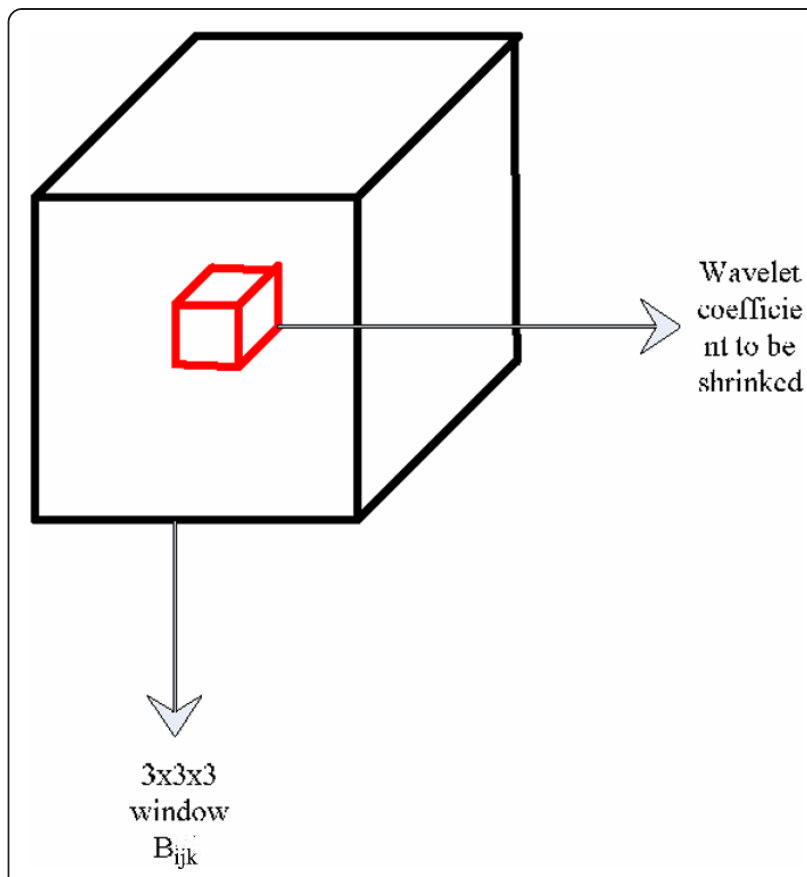

Figure 3 An illustration of the neighboring window centered at the wavelet coefficient to be shrinked. 
result of this may be the noise is reduced while the structural details important in medical image are blurred. As shown in a previous study [29], the oversmoothing could be compensated by exploiting the neighborhood statistics over a pixel to be denoised. By taking advantage of the essential feature of wavelet coefficients known as energy clustering within each subband, a 3D WNS method was proposed to preserve the structural information in MRI.

Let $W_{p q m}^{D_{i}}$ denotes the wavelet coefficient at location $(p, q, m)$ in subband $D^{i}$ which belongs to $\left\{L^{x} H^{y} H^{z}\right.$, $\left.H^{x} L^{y} H^{z}, H^{x} H^{y} L^{z}, H^{x} H^{y} H^{z}\right\}$, and $K^{L H H}, K^{H L H}, K^{H H L}, K^{H H H}$ represent different weights of different wavelet subbands, respectively, as shown in Figure 4. Weighting factors were determined according to the 3D Directional Filter Banks (3D DFB) developed by Lu and Do [30]. Then, $S_{i j k}^{D_{i}}$ will be calculated as follows:

$$
S_{i j k}^{D_{i}}=\left\{\begin{array}{l}
\sum_{(p, q, m) \in B_{i j k}} K^{L H H}\left(W_{p q m}^{D_{i}}\right)^{2} D_{i} \in L^{x} H^{\gamma} H^{z} \\
\sum_{(p, q, m) \in B_{i j k}} K^{H L H}\left(W_{p q m}^{D_{i}}\right)^{2} D_{i} \in H^{x} L^{\gamma} H^{z} \\
\sum_{(p, q, m) \in B_{i j k}} K^{H H L}\left(W_{p q m}^{D_{i}}\right)^{2} D_{i} \in H^{x} H^{\gamma} L^{z} \\
\sum_{(p, q, m) \in B_{i j k}} K^{H H H}\left(W_{p q m}^{D_{i}}\right)^{2} D_{i} \in H^{x} H^{\gamma} H^{z}
\end{array}\right.
$$

The estimator of the unknown noiseless coefficient $\widehat{\theta}_{i j k}$ is determined according to Equation (12).

\subsection{Algorithm summary}

The proposed 3D wavelet domain improved bilateral filter with the WNS thresholding (3DW-IBF) was summarized as follows (see Figure 5):

(1) Compute the square of noisy MR image $I$ to obtain its square magnitude $I_{\text {sq. }}$.

(2) Use 3D WT to get the approximation coefficients ( $\left.L^{x} L^{y} L^{z}, L^{x} L^{y} H^{z}, L^{x} H^{y} L^{z}, L^{x} L^{y} H^{z}\right)$ and the detail coefficients $\left(L^{x} H^{y} H^{z}, H^{x} L^{y} H^{z}, H^{x} H^{y} L^{z}, H^{x} H^{y} H^{z}\right)$ of $I_{\text {sq }}$ (see Section 2.2).

(3) The bias in approximation coefficients is removed by subtracting $4 \sigma_{n}^{2}[15]$ (see Section 2.1).

(4) These unbiased approximation coefficients are passed through the 3D improved bilateral filter (see Section 2.3).

(5) Denoise the detail coefficients using 3D WNS thresholding technique (see Section 2.4).

(6) Compute inverse 3D WT of the filtered approximation and the denoised detail coefficients to obtain the estimate of $I_{\text {sq }}$ (see Section 2.2).

(7) The square root of the resultant gives the denoised magnitude MR image.

\section{Experiments and results}

\subsection{Experimental data description}

We have carried out experiments with both simulated and real data. To conduct the experiments over synthetic data, three simulated MR images (T1, T2 and PD) with $1 \mathrm{~mm}^{3}$ voxel resolution (8-bit quantization) from the Brainweb phantom [31] were used. Each image contained $181 \times 217 \times 181$ voxels. To simulate Rician noise, we added zero mean Gaussian noise to the real and imaginary parts of the simulated MR data and afterwards the magnitude image was computed.

To evaluate the proposed approach on real clinical data, three datasets were used. Informed consent was obtained from all volunteers in accordance with our institution's policies regarding human subjects. The first dataset consisted of an MP-RAGE T1w volumetric sequence $(256 \times 240 \times 176$ voxels with a voxels resolution of $1 \mathrm{~mm}^{3}$ ) acquired on a Siemens $1.5 \mathrm{~T}$ Vision scanner. The acquisition parameters were $\mathrm{TR}=9 \mathrm{~ms}$, $\mathrm{TE}=$ $4 \mathrm{~ms}$, flip angle $=10^{\circ}$, TI $=2 \mathrm{~ms}$, TD $=200 \mathrm{~ms}$.

The second dataset was obtained with a TSE-FLAIR volumetric sequence $(256 \times 256 \times 160$ voxels with a voxels resolution of $0.94 \times 0.94 \times 1 \mathrm{~mm}^{3}$ ) acquired on a Philips Gyroscan 3 Tesla scanner (Best, Netherlands) using a sensitivity encoding (SENSE) acceleration factor of 2, TR $=14 \mathrm{~ms}$, and $\mathrm{TE}=140 \mathrm{~ms}$. Although parallel acquisition techniques such as SENSE or generalized autocalibrating partially parallel acquisitions introduce a spatially varying noise variance across the image, we used this dataset here to show the capability of the proposed approach on MR images with spatially varying noise.

Finally, we have obtained an image with a particularly large amount of noise by courtesy of Huiping Shi (Qiqihaer Medical College, Qiqihaer, China), as to assess the performance of the methods under extreme conditions. It was obtained on a 0.5 Tesla Neusoft-Philips, with parameters $\mathrm{TR}=20 \mathrm{~ms}, \mathrm{TE}=5 \mathrm{~ms}$, flip angle $=90^{\circ}$, field of view $=26 \mathrm{~cm}$, matrix $=256 \times 160$, slice thickness $=2$ $\mathrm{mm}$. The resulting $3 \mathrm{D}$ image has size $512 \times 512 \times 40$ voxels. Its original values are in the range $[0,255]$.

\subsection{Quantitative and qualitative metrics}

The efficiency of the denoising methods was compared quantitatively and qualitatively. For quantitative assessment, the mean squared error (MSE) and the Structural Similarity index (SSIM) [32] were evaluated. These measures were computed with the noise-free MR images as the ground truth. MSE is an objective measure that quantifies the deviation of estimated values from the true value

$$
\mathrm{MSE}=\frac{1}{M} \sum_{i=1}^{M}\left(I(i)-I_{0}(i)\right)^{2}
$$




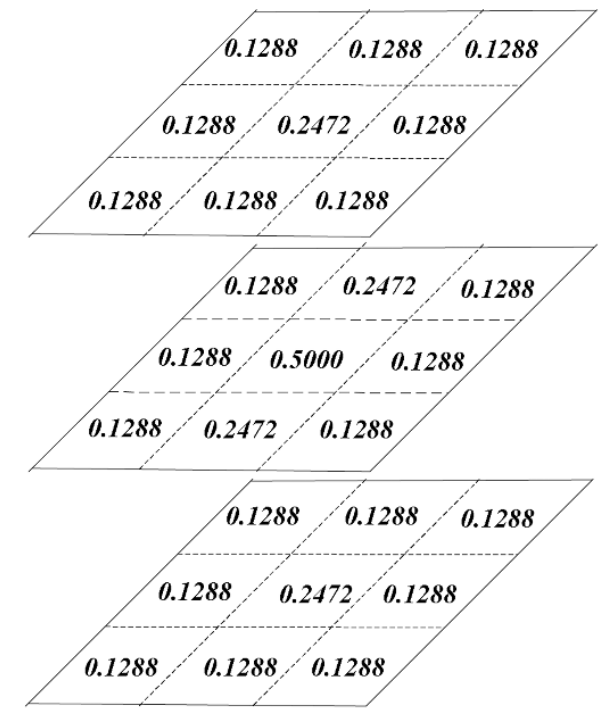

(a) $\mathbf{K}^{\mathrm{LHH}}$

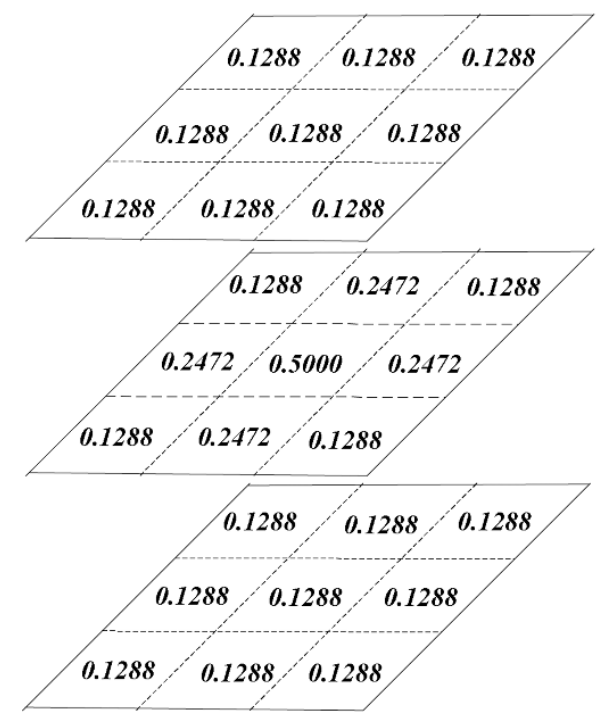

(c) $\mathrm{K}^{\mathrm{HHL}}$

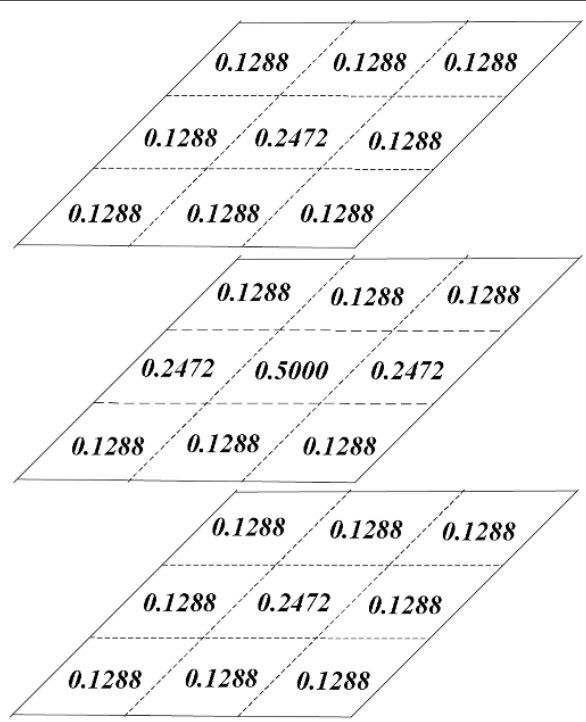

(b) $\mathbf{K}^{\mathrm{HLH}}$

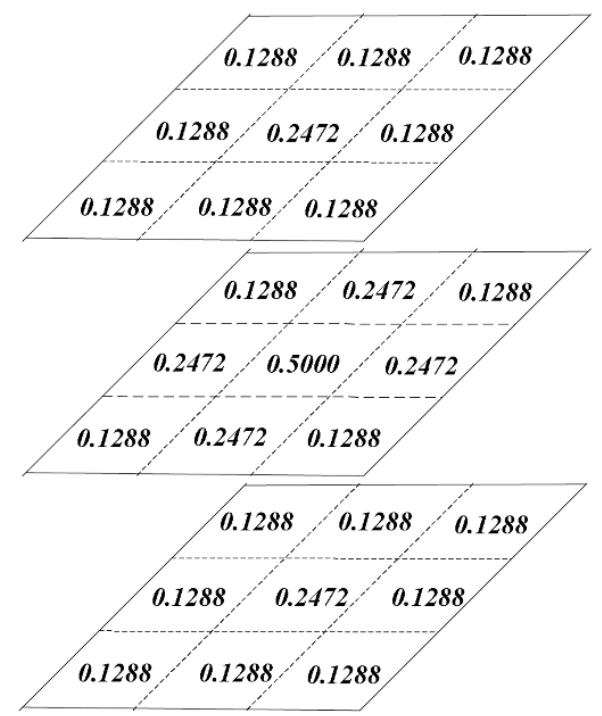

(d) $\mathbf{K}^{\mathrm{HHH}}$

Figure 4 Weighting coefficients of 3D WNS thresholding

where $I(i)$ and $I_{0}(i)$ are the pixel (voxel) values at position $i$ of the original image and the denoised image, respectively. $M$ denotes the number of the pixels in each image. MSE yields the same relative ordering among methods as the power signal-to-noise ratio (PSNR) and the root mean squared error (RMSE):

$$
\mathrm{PSNR}=10 \log _{10} \frac{255^{2}}{\mathrm{MSE}}
$$

$$
\mathrm{RMSE}=\sqrt{\mathrm{MSE}}
$$

Though, MSE is most commonly employed similarity metric, it is not optimal with respect to the perceived quality. Our second performance measure was the SSIM, which was employed to study the structural and perceptual similitude between the original and denoised images. SSIM is an effective alternative that improvizes the error measures and is also consistent 


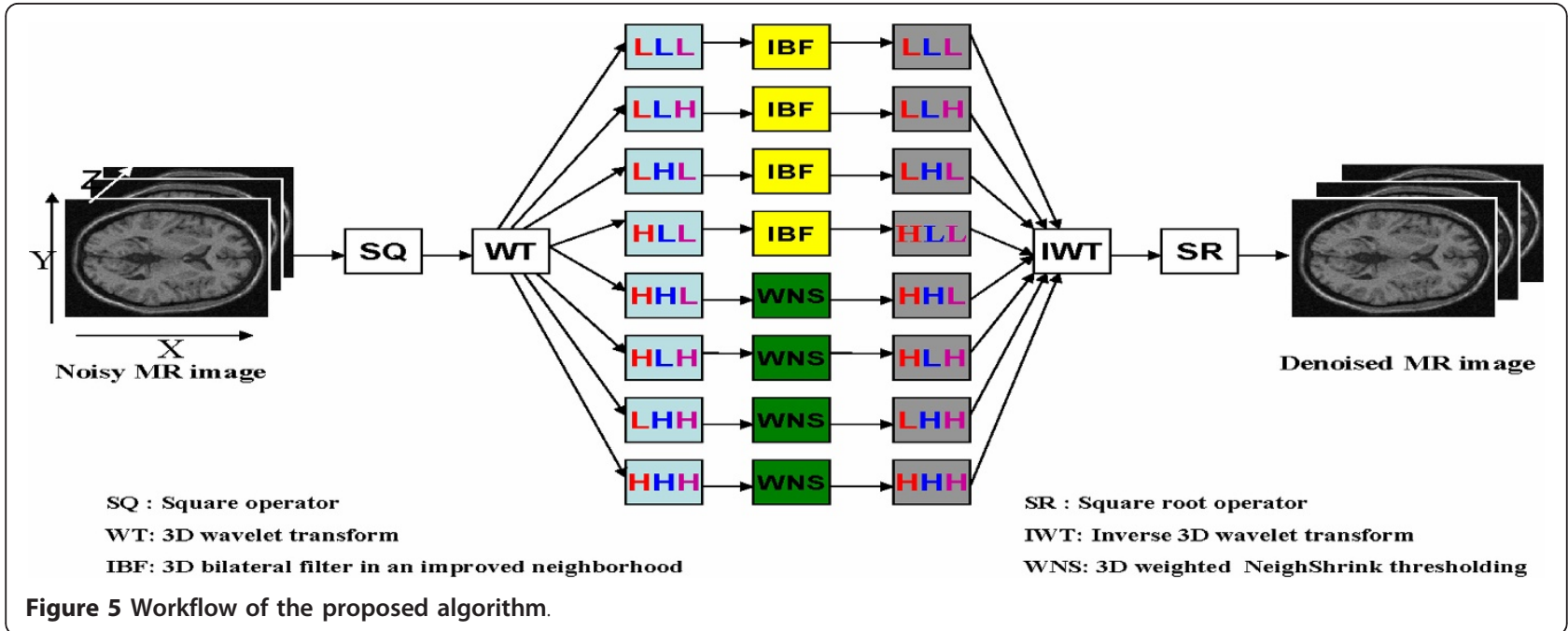

with the visual perception. As mentioned in previous section, MRI consists of delicate structural details, for which MSE is not sufficient to quantify the restored information. Therefore, SSIM is also used to study the structural and perceptual closeness between the denoised and the original images. The SSIM index is estimated locally over a $11 \times 11$ window, which moves pixel-by-pixel over the entire image. The final value of SSIM is the mean of SSIM index calculated over the $N$ local regions. The SSIM between the image $X$ and $Y$ is calculated from

$$
\operatorname{SSIM}(x, y)_{N}=\frac{\left(2 \mu_{x} \mu_{y}+c_{1}\right)\left(2 \delta_{x y}+c_{2}\right)}{\left(\mu_{x}^{2}+\mu_{y}^{2}+c_{1}\right)\left(\delta_{x}^{2}+\delta_{y}^{2}+c_{2}\right)}
$$

where $\mu$ is the mean intensity, $\delta$ denotes the standard deviation, and the constants $c_{1}=0.01, c_{2}=0.03$ were chosen as recommended in Wang et al. [32].

$$
\operatorname{SSIM}(X, Y)=\frac{1}{N} \sum_{R=1}^{N} \operatorname{SSIM}(x, y)_{R}
$$

The value of SSIM lies between $[-1,1]$. Alternatively, the SSIM can also be given in percentage (\%). Larger value of SSIM means high similarity between the compared images.

Visual assessment of the residual image was employed for qualitative evaluation. The residual image was obtained by subtracting the denoised image from the noisy image [12]. The residual image was required to verify the traces of anatomical information in clinical image removed during denoising. So, this could reveal the excessive smoothing and blurring of small structural details contained in the image.

\subsection{Validation on simulated dataset}

We have compared, qualitative and quantitatively the performance of our proposed algorithm with optimal estimated parameters $\left(R_{\text {neighbor }}=7, \delta_{d}=5, \delta_{r}=1.5 \sigma_{n}\right)$ with other three state-of-the-art filtering algorithms: the unbiased nonlocal means filter (UNLM) [12], the adaptive blockwise non-local means filter (ABONLM) [9], and the 2D wavelet domain bilateral filter (2DW-BF) [19].

Our algorithm was quantitatively compared, using the synthetic data referred in Section 3.1. The values of MSE and SSIM obtained for the synthetic data using the aforementioned denoising techniques were tabulated in Tables 1 and 2, respectively. The performance of 3DWIBF with weighted Neighshrink is better than UNLM, ABONLM, and 2DW-BF with respect to the quantitative metrics. Lower value of MSE and higher value of SSIM showed that our method significantly outperforms others.

The results on T1-weighted images were shown in Figure 6. The original MR image (Figure 6a) and the noisy image with $5 \%$ Rician noise added (Figure $6 \mathrm{~b}$ ) were presented. Some structural details were smoothed in the results obtained by the UNLM filter (Figure 6c). While the UNLM filter could preserve the distinct edge features, it failed to preserve small structural details. The results obtained by the ABONLM filter (Figure 6d) were better than those by the UNLM filter. However, some regions were blurred and some useful information was lost. We found that the 2D-WBF filter has the coarse edge effect (Figure 6e) compared to our proposed filter (Figure 6f).

The results on T2-weighted images with 7\% Rician noise added were shown in Figure 7 . The result 
Table 1 Comparisons of experimental results in MSE

\begin{tabular}{|c|c|c|c|c|c|c|c|c|c|c|c|c|c|c|c|}
\hline \multirow[t]{3}{*}{ Algorithm } & \multicolumn{15}{|c|}{ Test image and noise level } \\
\hline & \multicolumn{5}{|c|}{ T1-weighted MR image } & \multicolumn{5}{|c|}{ T2-weighted MR image } & \multicolumn{5}{|c|}{ PD-weighted MR image } \\
\hline & $1 \%$ & $3 \%$ & $5 \%$ & $7 \%$ & $9 \%$ & $1 \%$ & $3 \%$ & $5 \%$ & $7 \%$ & $9 \%$ & $1 \%$ & $3 \%$ & $5 \%$ & $7 \%$ & $9 \%$ \\
\hline UNML & 2.50 & 11.42 & 24.71 & 43.69 & 68.12 & 3.31 & 18.62 & 38.68 & 64.97 & 97.78 & 2.68 & 13.85 & 29.52 & 48.78 & 71.20 \\
\hline ABONLM & 3.28 & 19.78 & 51.07 & 97.98 & 161.39 & 6.55 & 24.76 & 56.19 & 101.03 & 159.83 & 4.01 & 18.82 & 46.08 & 87.25 & 141.02 \\
\hline 2DW-BF & 3.08 & 11.56 & 26.06 & 51.56 & 62.26 & 7.23 & 20.33 & 57.30 & 68.27 & 90.03 & 3.65 & 15.19 & 30.63 & 43.67 & 62.77 \\
\hline 3DW-IBF & 2.07 & 8.20 & 15.11 & 22.31 & 29.58 & 4.62 & 15.54 & 29.21 & 50.10 & 67.38 & 3.52 & 11.18 & 20.65 & 26.12 & 41.58 \\
\hline
\end{tabular}

Table 2 Comparisons of experimental results in SSIM

\begin{tabular}{|c|c|c|c|c|c|c|c|c|c|c|c|c|c|c|c|}
\hline \multirow[t]{3}{*}{ Algorithm } & \multicolumn{15}{|c|}{ Test image and noise level } \\
\hline & \multicolumn{5}{|c|}{ T1-weighted MR image } & \multicolumn{5}{|c|}{ T2-weighted MR image } & \multicolumn{5}{|c|}{ PD-weighted MR image } \\
\hline & $1 \%$ & $3 \%$ & $5 \%$ & $7 \%$ & $9 \%$ & $1 \%$ & $3 \%$ & $5 \%$ & $7 \%$ & $9 \%$ & $1 \%$ & $3 \%$ & $5 \%$ & $7 \%$ & $9 \%$ \\
\hline UNML & 0.984 & 0.926 & 0.869 & 0.814 & 0.763 & 0.987 & 0.941 & 0.895 & 0.852 & 0.814 & 0.985 & 0.934 & 0.880 & 0.829 & 0.783 \\
\hline ABONLM & 0.943 & 0.862 & 0.827 & 0.798 & 0.770 & 0.967 & 0.902 & 0.865 & 0.839 & 0.816 & 0.968 & 0.897 & 0.855 & 0.823 & 0.796 \\
\hline 2DW-BF & 0.967 & 0.929 & 0.931 & 0.896 & 0.873 & 0.991 & 0.948 & 0.899 & 0.867 & 0.856 & 0.997 & 0.930 & 0.881 & 0.836 & 0.830 \\
\hline 3DW-IBF & 0.989 & 0.934 & 0.921 & 0.913 & 0.908 & 0.989 & 0.942 & 0.916 & 0.901 & 0.882 & 0.988 & 0.946 & 0.903 & 0.890 & 0.855 \\
\hline
\end{tabular}

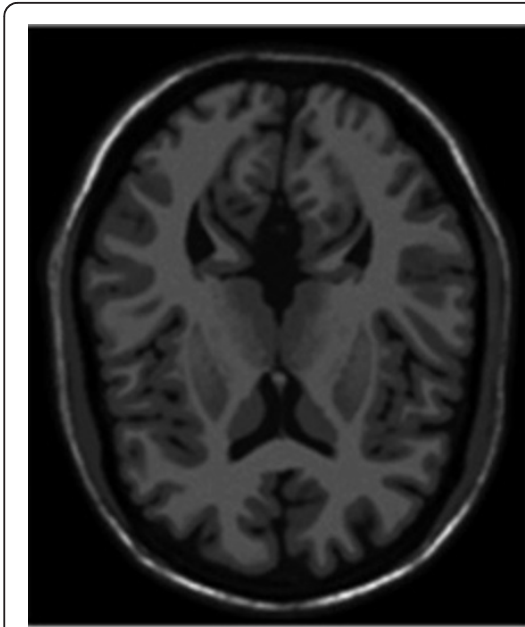

a

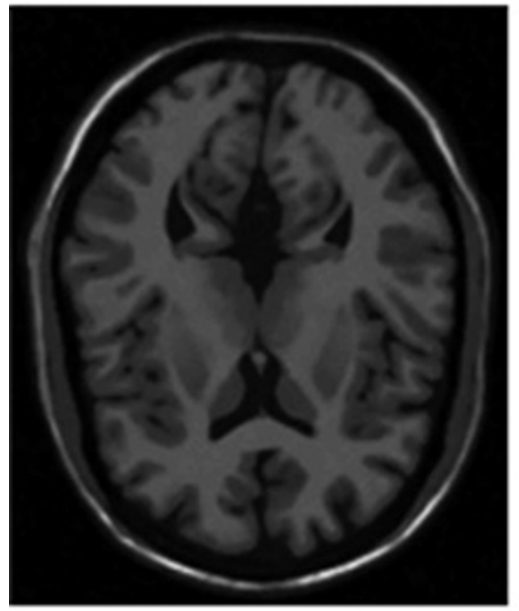

d

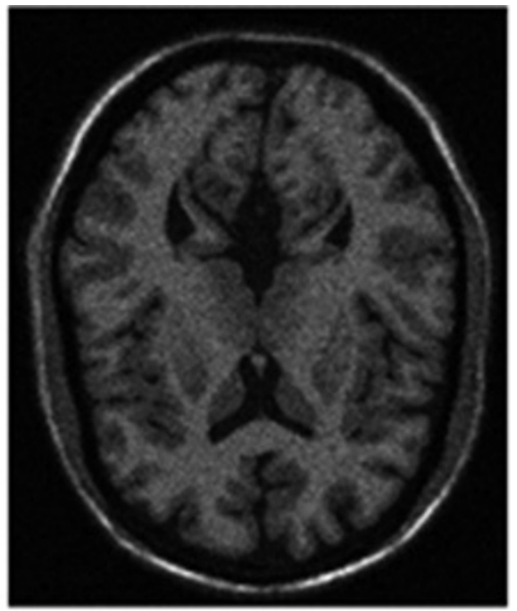

b

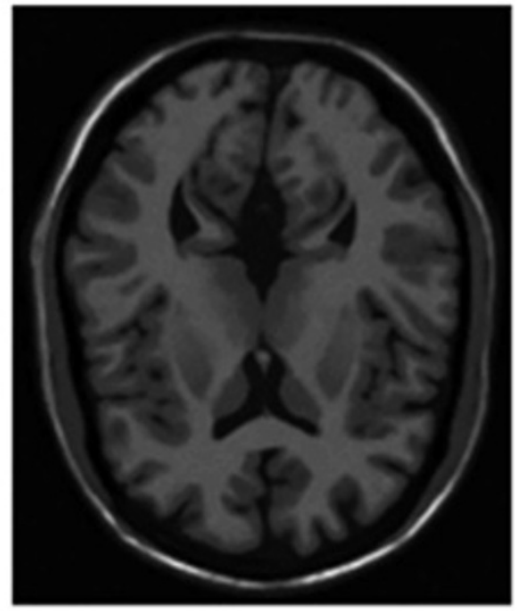

e

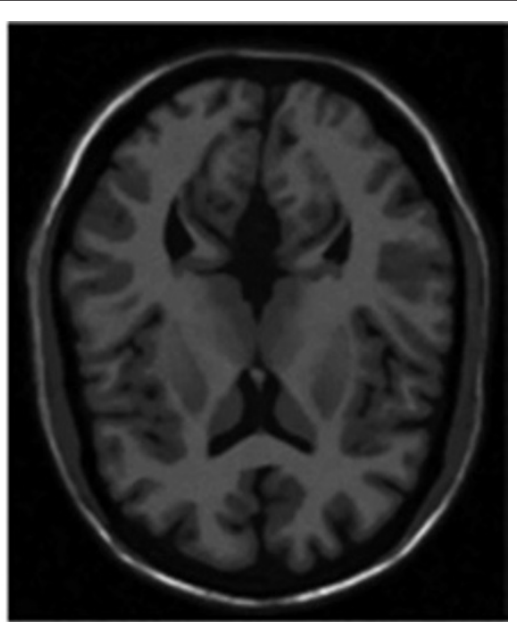

c

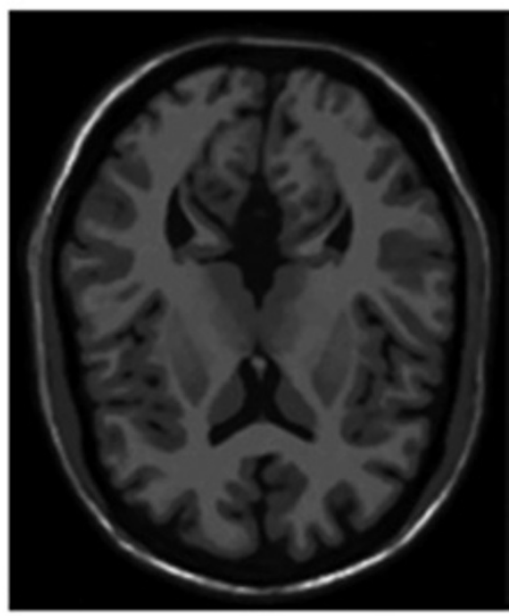

f

Figure 6 Comparison of experiment results on T1-weighted. (a) The original MR image, (b) 5\% Rician noise added, (c) results of the UNLM filter, (d) results of the ABONLM filter, (e) results of the 2DW-BF filter, (f) results of the 3DW-IBF filter. 
obtained by the 2DW-BF filter (Figure 7e) reserved more information than the result obtained by the UNLM filter (Figure 7c) and that obtained by the ABONLM filter (Figure 7d). However, the 2DW-BF filter smoothed the homogeneous regions. We observed that the result obtained by our proposed filter (Figure $7 \mathrm{f}$ ) could recover as much information as that by the 2DW-BF filter.

The results on PD-weighted image were shown in Figure 8 . The noisy image with $9 \%$ Rician noise added was presented (Figure $8 \mathrm{~b}$ ). We found that the interface between gray and white matter in the result by the UNLM filter (Figure 8c) was away from that of the original image (Figure 8a), and the result by the ABONLM filter (Figure 8d) was oversmoothed. There was still some obvious noise in the result by the 2DW-BF filter (Figure 8e). Both the small structures and the boundaries were preserved well by the 3DW-IBF filter (Figure 8f).

\subsection{Validation on clinical dataset}

Since the original images already have noise, neither ideal residual image nor quantitative results can be obtained. The denoising results obtained for the T1weighted brain image were shown in Figure 9. The residual images of the UNLM filter (Figure 9c), the ABONLM filter (Figure 9e), and the 3DW-IBF method (Figure 9i) did not reveal significant anatomical information. The residual image of the 2DW-BF filter showed the extent of smoothing along the edges of the image (Figure 9g). While the 2DW-BF filter could preserve the distinct edge features, it blurs the heterogeneous regions and hence, reduces the contrast between the gray and the white matter regions. The results of filtering SENSE

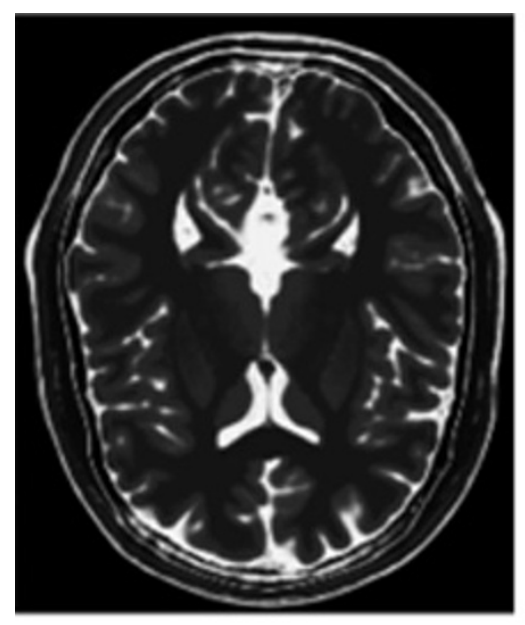

a

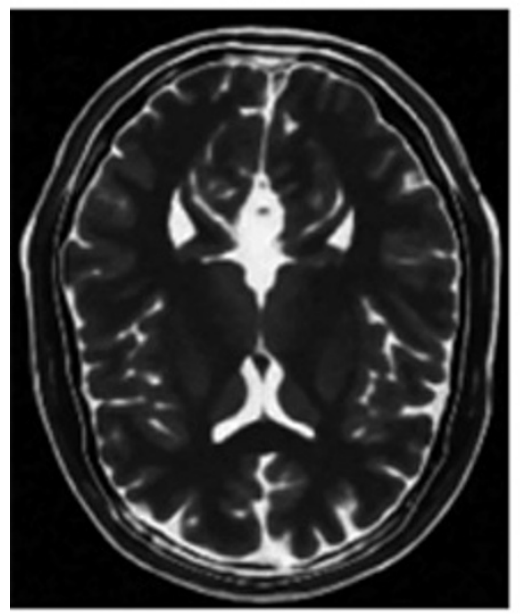

d

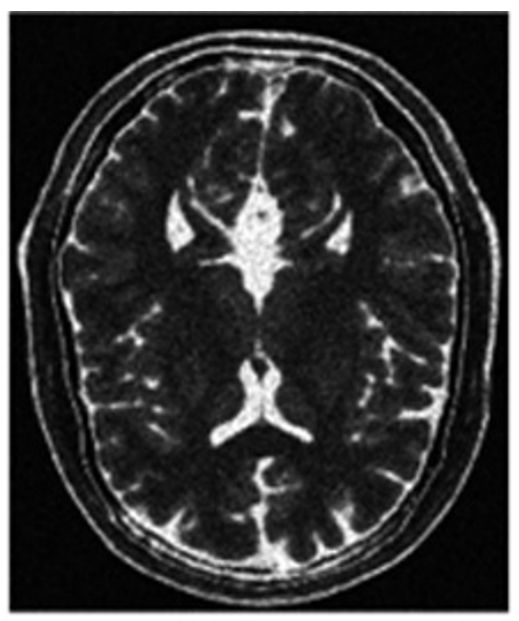

b

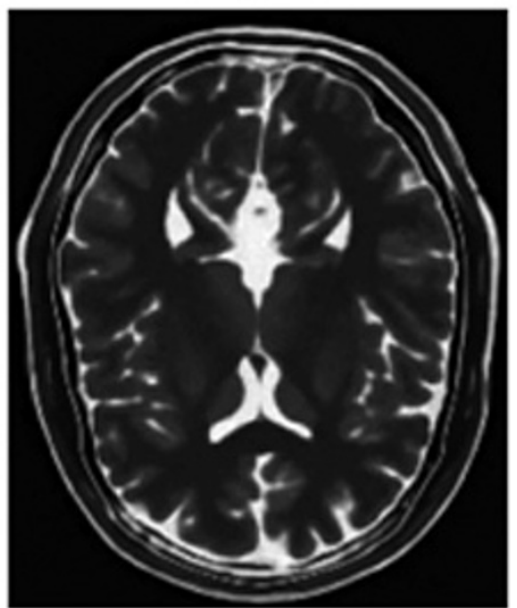

e

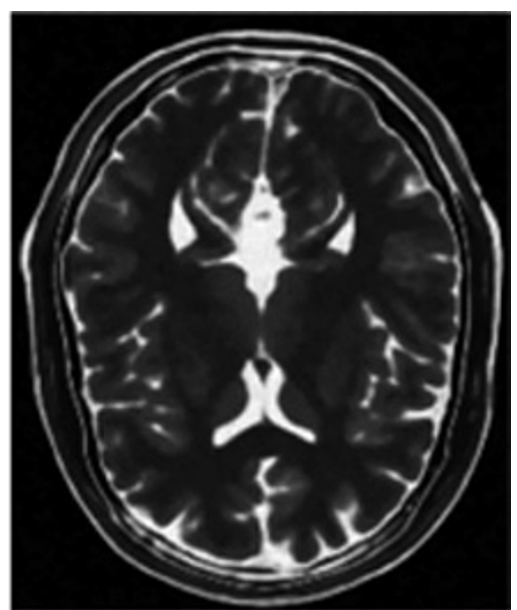

c

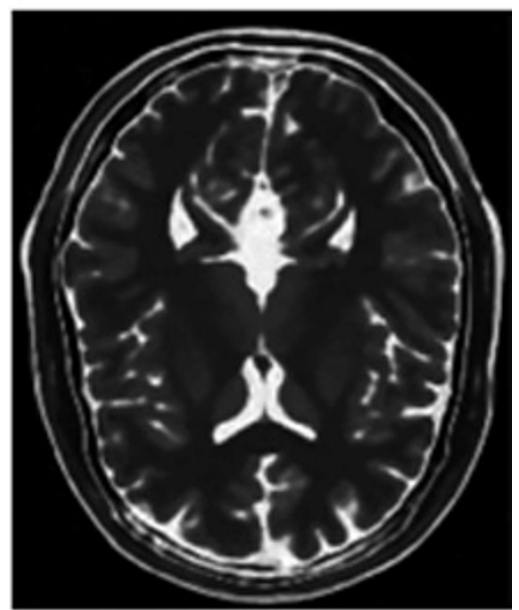

f

Figure 7 Comparison of experiment results on T2-weighted. (a) The original MR image, (b) 7\% Rician noise added, (c) results of the UNLM filter, (d) results of the ABONLM filter, (e) results of the 2DW-BF filter, (f) results of the 3DW-IBF filter. 


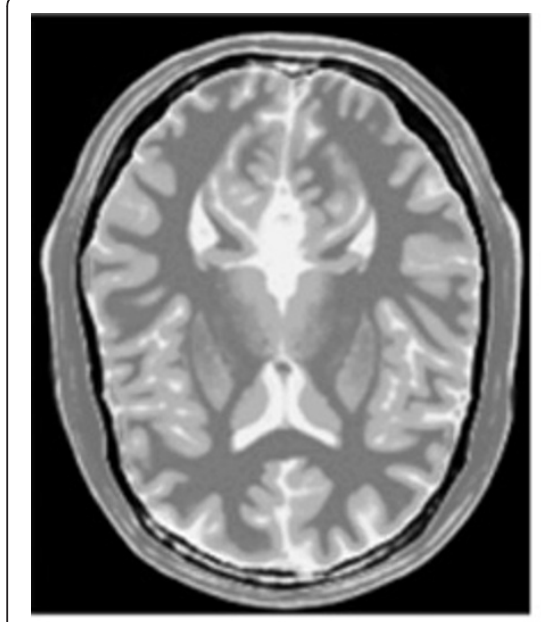

a

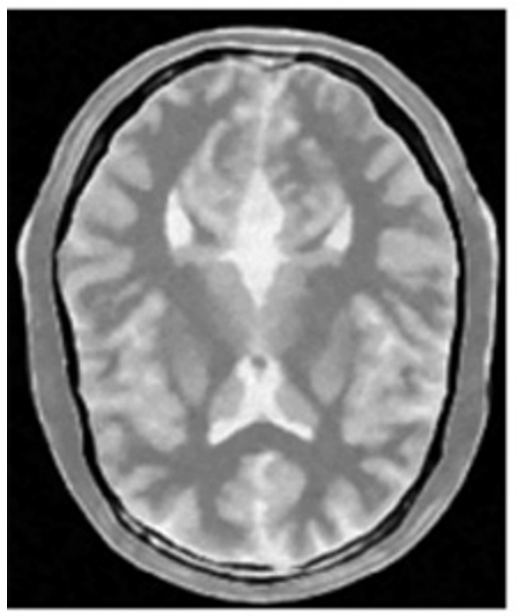

d

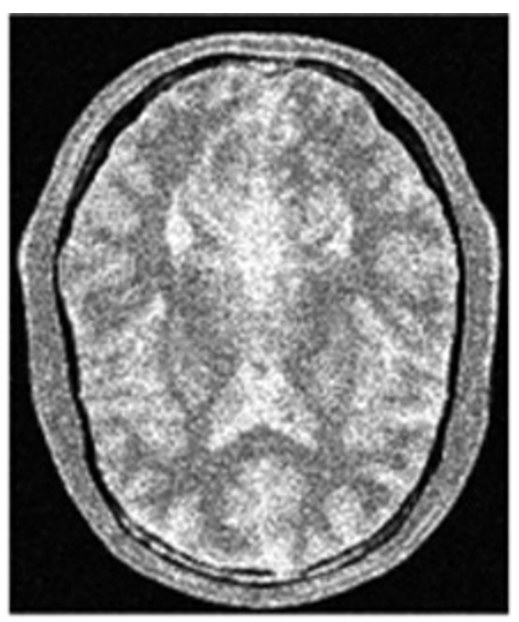

b

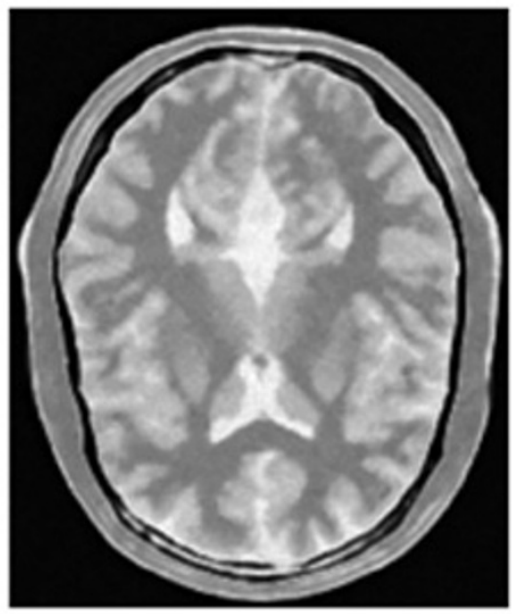

e

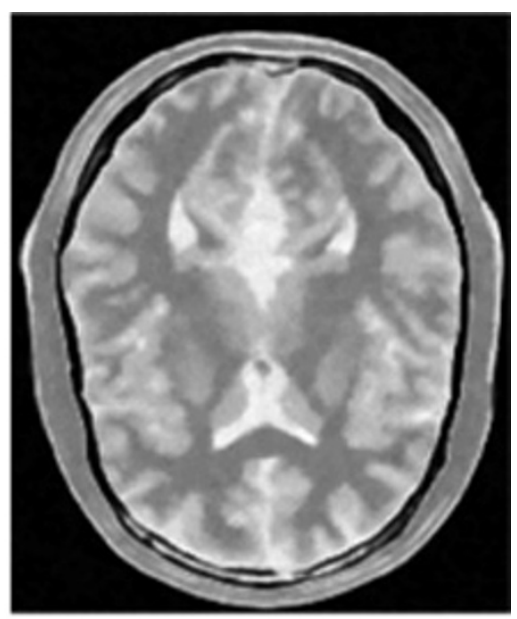

c

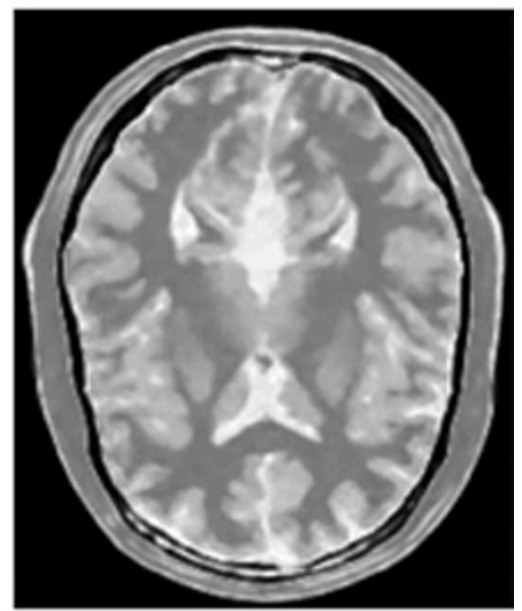

f

Figure $\mathbf{8}$ Comparison of experiment results on PD-weighted. (a) The original MR image, (b) 9\% Rician noise added, (c) results of the UNLM filter, (d) results of the ABONLM filter, (e) results of the 2DW-BF filter, (f) results of the 3DW-IBF filter.

images reconstructed from patient brain data were shown in Figure 10. The ULM filter and the 2DW-BF method left unfiltered noise in the image (Figure 10b, f). With the ABONLM method, the edges were blurred, reducing the image sharpness (Figure 10d). Alternatively, with the 3DW-IBF method, the edges were preserved well and the contrast between the gray and the white tissues were also preserved well (Figure 10h). The results obtained for the T1-weighted brain image with heavy noise were shown in Figure 11. With the 2DW$\mathrm{BF}$ method, the sharpness along the edges was smoothed (Figure 11f). Comparing the results of the ULM filter (Figure 11b) and the ABONLM method (Figure 11d), it was evident that the 3DW-IBF method preserves well the details (Figure 11h).

\section{Discussion and conclusion}

The sources that introduce uncertainty in voxel intensity are many and are generally derived from one of two categories: thermal noise and physiological noise. Other sources may also exist in the electronics of the acquisition system, such as digitization, but these can be minimized in an ideal condition. Thermal noise is usually considered as "white noise" because it is expected that its power should be equal for all frequencies within the readout bandwidth. Because MR images are reconstructed using the Fourier transform, the variance that characterizes the uncertainty due to thermal noise is constant throughout the imaging volume. But the physiological noise differs. In our study, we could only estimate the variance that characterizes the uncertainty of 


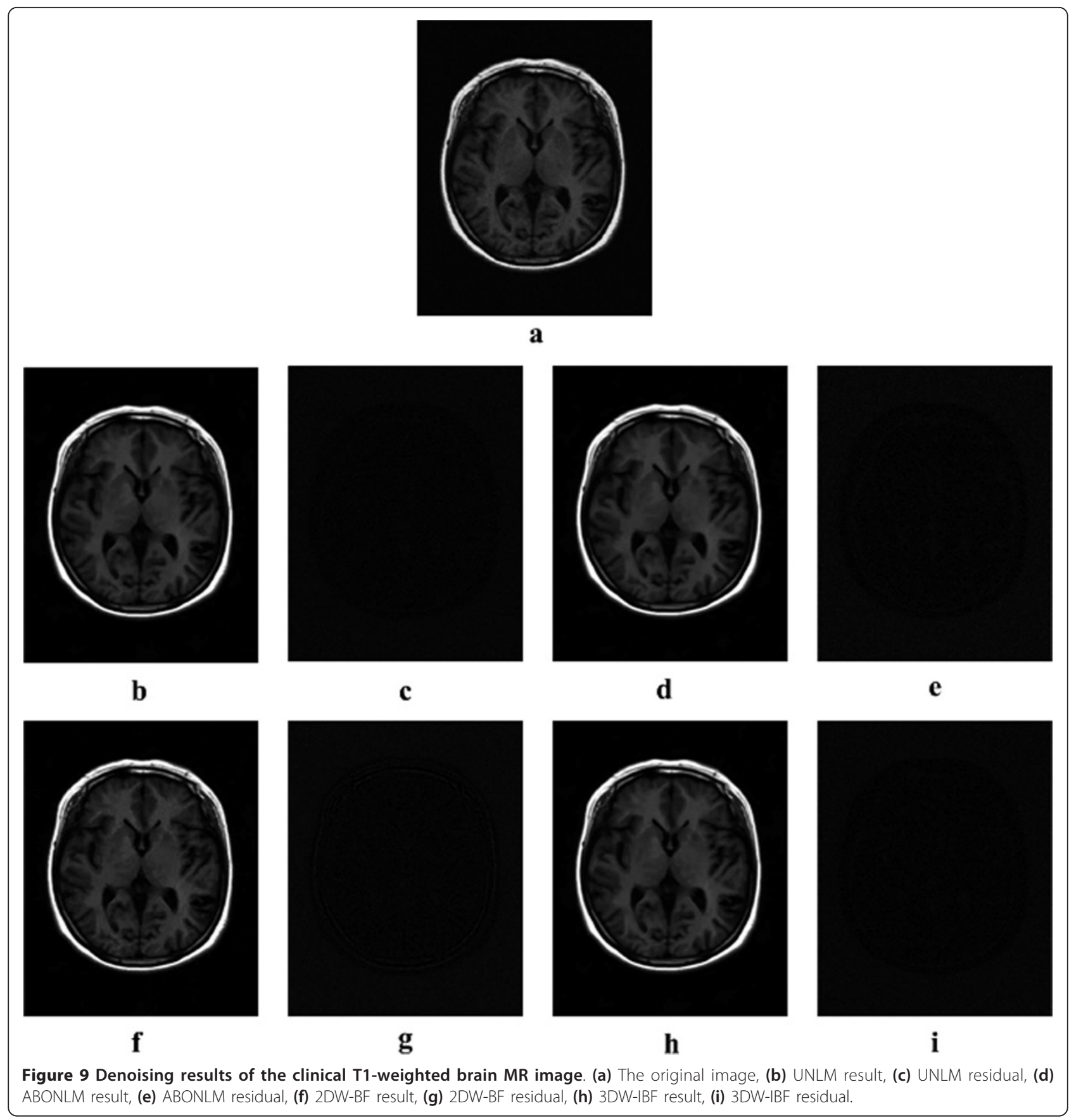

the MR measurement due to thermal noise. But we expected to estimate the variance component due to physiological noise such as flow, MR spin history effect. To this end, it requires many repeated acquisitions. It would also be difficult to isolate the variance due to patient motion in such repeated measurements (which is something the registration step is actually trying to diminish). Therefore, we did not estimate the noise introduced by physiological effects.
In this study, we proposed a $3 \mathrm{D}$ extension of the wavelet domain bilateral filtering ideas for Rician noise removal. Due to the delineating capability of wavelet, 3D WT was employed to decompose the MR image into the approximation and the detailed subbands. Compared to $2 \mathrm{D} \mathrm{WT}$, the inherent advantages of $3 \mathrm{D} \mathrm{WT}$ is apparent due to improved ability to model "through-plane" structure. 2D denoising ignores through-plane signal correlations; each slice is treated independently. 


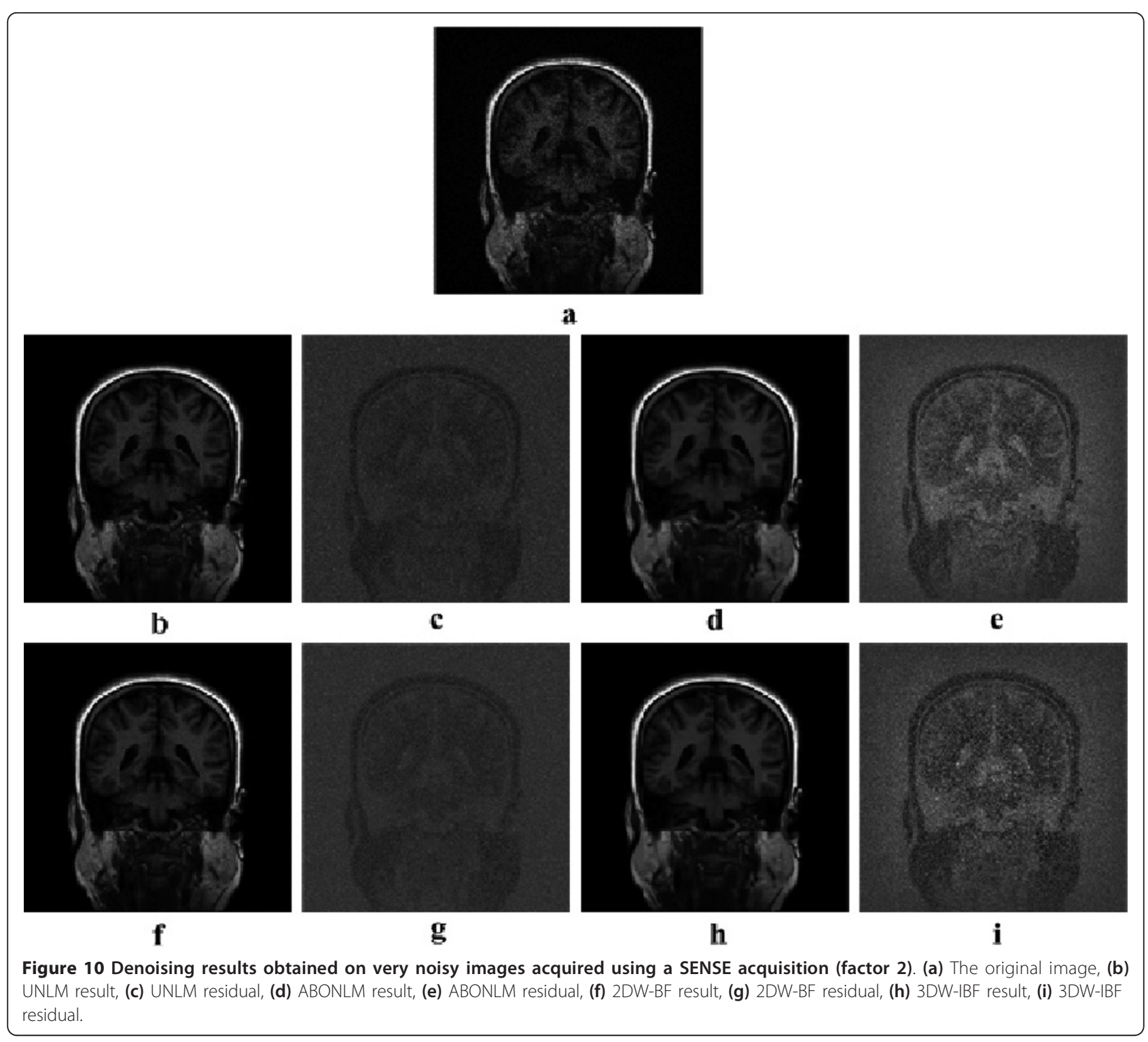

Consider a slender fiber tract. If the imaging plane is orthogonal to the fiber axis, it would be much more difficult to distinguish the "structure" from spurious, bright, noise pixel. 3D denoising uses structural correlations from all three principle planes, and is robust to fiber axis orientation.

Considering the similarities in the wavelet domain where the data and noise can be efficiently discriminated, 3D bilateral filtering of the approximation coefficients in a modified neighborhood eliminates the higher magnitude noise components carried into the approximation subbands. Utilizing a group of a square and a line as neighbor to replace the original cubic neighbor for weight estimation improves the computation accuracy. Noticing the fact that the wavelet subband coefficients of different orientations have different properties of energy clustering, a WNS thresholding has been proposed to threshold the noisy coefficients in the detailed subbands. Exploiting the interscale dependencies among the detailed coefficients tends to improve the performance of wavelet thresholding, and thus, it also enhances the denoising efficiency for MR images with spatially varying noise. In summary, the utilization of neighborhood similarities using wavelet domain bilateral filter and Neighshrink improves the noise cancellation efficiency and preserves the structural information effectively.

Experiments were carried out on both simulated and real datasets. Quantitative results using two different quality measures show a better behavior of the proposed scheme when compared to other state-of-the-art filters for different noise levels. 


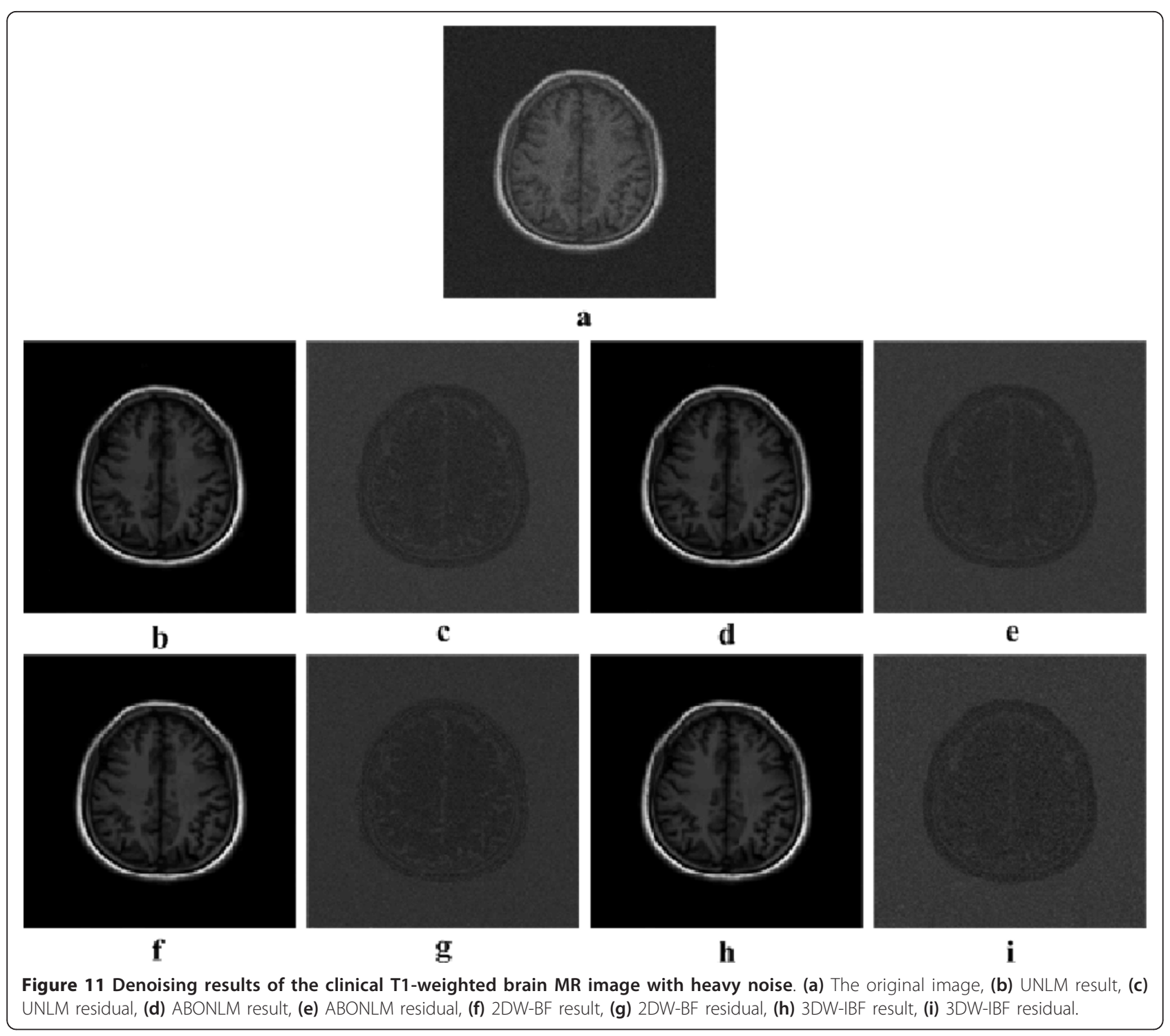

An ideal filter for MR images with spatially varying noise levels must be able to improve the image PSNR while preserving image important structures and avoiding the generation of artifacts. The results indicated that parameter $\sigma_{n}$ chosen as a constant in all image areas may lead to the enhancement of noise-generated gradients in high-noise areas that could be incorrectly identified as anatomical structures of different tissue type characteristics for the imaging modality such as vessels. Therefore, it is necessary to derive a strategy that optimizes the choice of $\sigma_{n}$ with respect to the local characteristics of the considered neighborhoods. Hence, this study can be extended to make the choice of $\sigma_{n}$ locally adaptive, optimizing the denoising procedure for all the noise levels.

\section{Acknowledgements}

We would like to thank McConnell Brain Imaging Center (BIC) of the Montreal Neurological Institute, McGill University, for providing access to the MR data in the BrainWeb Database (http://www.bic.mni.mcgill.ca/brainweb). We would also like to thank VisAGeS of the IRISA Institute, University of Rennes I, for providing online applications (http://www.irisa.fr/visages/ benchmarks/).

\section{Author details}

'Department of Computational Mathematics, Jilin University, Chang chun

China ${ }^{2}$ Department of Clinical Medicine, Qiqihaer Medical College, Qiqihaer, China

\section{Competing interests}

The authors declare that they have no competing interests.

Received: 20 July 2011 Accepted: 21 February 2012

Published: 21 February 2012 


\section{References}

1. G McGibney, MR Smith, An unbiased signal-to-noise ratio measure for magnetic resonance images. Med Phys. 20(4), 1077-1078 (1993). doi:10.1118/1.597004

2. J Sijbers, AJ Dekker, Maximum likelihood estimation of signal amplitude and noise variance from MR data. Magn Reson Med. 51(3), 586-594 (2004). doi:10.1002/mrm.10728

3. P Perona, J Malik, Scale-space and edge detection using anisotropic diffusion. IEEE Trans Pattern Anal Mach Intell. 12(7), 629-639 (1990). doi:10.1109/34.56205

4. G Gerig, O Kubler, R Kikinis, FA Jolesz, Nonlinear anisotropic filtering of MRI data. IEEE Trans Med Imag. 11(2), 221-232 (1992). doi:10.1109/42.141646

5. AA Samsonov, CR Johnson, Noise-adaptive nonlinear diffusion filtering of MR images with spatially varying noise levels. Magn Reson Med. 52(4), 798-806 (2004). doi:10.1002/mrm.20207

6. K Krissianand, S Aja-Fernández, Noise-driven anisotropic diffusion of noisy MRI data. IEEE Trans Image Process. 18(10), 2265-2274 (2009)

7. M Martin-Fernandez, C Alberola-Lopez, J Ruiz-Alzola, CF Westin, Sequentia anisotropic Wiener filtering applied to 3D MRI data. Magn Reson Imag. 25(2), 278-292 (2007). doi:10.1016/.mri.2006.05.001

8. P Coupé, P Yger, S Prima, P Hellier, C Kervrann, C Barillot, An optimized blockwise non local means denoising filter for 3D magnetic resonance images. IEEE Trans Med Imag. 27(4), 425-441 (2008)

9. JV Manjón, P Coupé, L Martí-Bonmatí, DL Collins, M Robles, Adaptive nonlocal means denoising of MR images with spatially varying noise levels. J Magn Reson Imag. 31(1), 192-203 (2010). doi:10.1002/jmri.22003

10. A Buades, B Coll, J-M Morel, A non-local algorithm for image denoising, in CVPR 2005, San Diego, USA, 60-65 (June 2005)

11. N Wiest-Daesslé, S Prima, P Coupé, SP Morrissey, C Barillot, Rician noise removal by non-local means filtering for low signal-to-noise ratio MRI: applications to DT-MRI, in MICCAI 2008, New York, USA, 104-117 (September 2008)

12. JV Manjón, J Carbonell-Caballero, JJ Lull, G García-Martí, L Martí-Bonmatí, M Robles, MRI denoising using non-local means. Med Image Anal. 12(4), 514-523 (2008). doi:10.1016/j.media.2008.02.004

13. AM Wink, JBTM Roerdink, Denoising functional MR images: a comparison of wavelet denoising and Gaussian smoothing. IEEE Trans Med Imag. 23, 374-387 (2004). doi:10.1109/TMI.2004.824234

14. A Pizurica, AM Wink, E Vansteenkiste, W Philips, J Roerdink, A review of wavelet denoising in MRI and ultrasound brain imaging. Curr Med Imag Rev. 2(2), 247-260 (2006). doi:10.2174/157340506776930665

15. RD Nowak, Wavelet-based Rician noise removal for magnetic. IEEE Trans Image Process. 8(10), 1408-1419 (1999). doi:10.1109/83.791966

16. $\mathrm{P}$ Bao, L Zhang, Noise reduction for magnetic resonance images via adaptive multiscale products thresholding. IEEE Trans Med Imag. 22(9), 1089-1099 (2003). doi:10.1109/TMI.2003.816958

17. S Zaroubi, G Goelman, Complex denoising of MR data via wavelet analysis: application for functional MRI. Magn Reson Imag. 18(1), 59-68 (2000). doi:10.1016/S0730-725X(99)00100-9

18. ZQ Wu, JA Ware, J Jiang, Wavelet-based Rayleigh background removal in MRI. Electron Lett. 39(7), 603-605 (2003). doi:10.1049/el:20030396

19. CS Anand, JS Sahambi, Wavelet domain non-linear filtering for MRI denoising. Magn Reson Med. 28(6), 842-861 (2010)

20. X Yang, B Fei, A wavelet multiscale denoising algorithm for magnetic resonance (MR) images. Meas Sci Technol. 22, 025803 (2011). doi:10.1088/ 0957-0233/22/2/025803

21. H Gudbjartsson, S Patz, The Rician distribution of noisy MRI data. Magn Reson Med. 34, 910-914 (1995). doi:10.1002/mrm.1910340618

22. S Aja-Fernández, C Alberola-López, CF Westin, Noise and signal estimation in magnitude MRI and Rician distributed images: a LMMSE approach. IEEE Trans Image Process. 17(8), 383-398 (2008)

23. M Brummer, R Mersereau, R Eisner, R Lewine, Automatic detection of brain contours in MRI data sets. IEEE Trans Med Imag. 2(29), 153-166 (1993)

24. J Sijbers, D Poot, AJ den Dekker, W Pintjens, Automatic estimation of the noise variance from the histogram of a magnetic resonance image. Phys Med Biol. 52, 1335-1348 (2007). doi:10.1088/0031-9155/52/5/009

25. J Sijbers, AJ den Dekker, J Van Audekerke, M Verhoye, D Van Dyck, Estimation of the noise in magnitude MR images. Magn Reson Imag. 1(16), 87-90 (1998)
26. Z Chen, R Ning, Breast volume denoising and noise characterization by 3D wavelet transform. Comput Med Imag Graph. 28, 235-246 (2004). doi:10.1016/j.compmedimag.2004.04.004

27. C Tomasi, R Manduchi, Bilateral filtering for gray and color images, in ICCV 1998, Bombay, India, 839-846 (January 1998)

28. GY Chen, TD Bui, A Krzyzak, Image denoising with neighbour dependency and customized wavelet and threshold. Pattern Recogn. 38, 115-124 (2005). doi:10.1016/j.patcog.2004.05.009

29. D Zhou, W Cheng, Image denoising with an optimal threshold and neighbouring window. Pattern Recogn Lett. 29(11), 1694-1697 (2008). doi:10.1016/j.patrec.2008.04.014

30. Y Lu, MN Do, Multidimensional directional filter banks and surfacelets. IEEE Trans Image Process. 16(4), 918-931 (2007)

31. DL Collins, AP Zijdenbos, V Kollokian, JG Sled, NJ Kabani, CJ Holmes, AC Evans, Design and construction of a realistic digital brain phantom. IEEE Trans Med Imag. 17(3), 463-468 (1998). doi:10.1109/42.712135

32. Z Wang, AC Bovik, HR Sheikh, EP Simoncelli, Image quality assessment: from error visibility to structural similarity. IEEE Trans Image Process. 13(4), 600-612 (2004). doi:10.1109/TIP.2003.819861

doi:10.1186/1687-6180-2012-40

Cite this article as: Wang et al: Nonlinear filtering based on 3D wavelet transform for MRI denoising. EURASIP Journal on Advances in Signal

Processing 2012 2012:40.

\section{Submit your manuscript to a SpringerOpen ${ }^{\circ}$ journal and benefit from:}

- Convenient online submission

- Rigorous peer review

- Immediate publication on acceptance

- Open access: articles freely available online

- High visibility within the field

- Retaining the copyright to your article

Submit your next manuscript at $\gg$ springeropen.com 